\title{
Reply to reply to S-ICD: Is it time for radiological follow-ups? by Bisignani A et al.
}

\author{
Zefferino Palamà ${ }^{1}$ Giulia $\mathrm{My}^{1}$, Luigi $\mathrm{My}^{2}$, and Mariano Rillo \\ ${ }^{1}$ Casa di Cura Villa Verde Srl \\ ${ }^{2}$ Casa Di Cura Villa Verde
}

November 29, 2021

\begin{abstract}
Please let me know if it is necessary... we are working on a reply to a previous work
\end{abstract}

Reply to reply to S-ICD: Is it time for radiological follow-ups? by Bisignani A et al.

Zefferino Palamà ${ }^{1-2}$, MD, Giulia $\mathrm{My}^{3}, \mathrm{MD}$, Luigi $\mathrm{My}^{3}, \mathrm{MD}$, Mariano Rillo ${ }^{1}$, MD

1. Electrophysiology Service, Casa di Cura "Villa Verde", Taranto, Italy

2. Department of Life, Healt and Enviromenal Sciences, University of l'Aquila, L'Aquila, Italy

3. Cardiology Unit, Casa di Cura "Villa Verde", Taranto, Italy

Corresponding author:

Zefferino Palamà

Casa di Cura "Villa Verde"-Taranto, Italy

Via Golfo di Taranto, 22- Taranto

Email:zefferino.palama@icloud.com

\section{DISCLOSURES}

No external funding was received. The authors report no conflict of interest.

We thank Antonio Bisignani et al. for their valuable contribution regarding the evaluation of the case reported by us ${ }^{1}$.

Despite the results of their experience regarding the stability of the subcutaneous catheter even with the technique with only two incisions, in our opinion perhaps 38 cases, especially without considering selective patients, could be not sufficient to exclude the risk of mid and long-term lead migration. We think that some groups of patients can be a challenge during perioperative management and follow-up. Furthermore, we know that some special categories of patients, such as athletes, for examples cyclists, can face sensing mistakes due to the position and that they need an optimal programmation to avoid inappropriate shock ${ }^{2}$. Leads displacement can occur in every kind of implantable device, even a transvenous one; and this complication is more frequent in young and athletic patients, but the literature doesn't give us any suggestion on the kind of ideal device based on specific physical activity ${ }^{3}$. S-ICD is very safe in detecting and treating fatal $\operatorname{arrhythmias}^{4}$; anyway, follow-up electric data don't match with the possibility of lead displacement, while this is possible with a transvenous defibrillator. 
In the case proposed by us, the effectiveness of the shock was not verified before lead reimplantation because we considered it ethically incorrect. Nevertheless, a lead displacement can lay the foundation for shock failure. That is a real and disastrous occurrence that, to date, only an X-ray check can reveal. The importance that an unknown displacement can have, especially possible in some categories of patients at risk of developing this complication, such as the obese, young patients, or athletes, justifies the efforts to exclude it and has prompted us to suggest routine X-ray checks. Only in this way is it possible to quantify a problem that is currently unknown. The hope is that by interrogating the device it is possible to obtain technical parameters that can make the displacement of the lead manifest, as is the case with a transvenous devices.

\section{REFERENCES}

1. Rillo M, Palamà Z, My G, Punzi R, Aurelio A, Aloisio A, Giannattasio C, My L. S-ICD: Is it time for radiological follow-ups? J Cardiovasc Electrophysiol. 2021;32:2752-2754.

2. Sciarra L, Nesti M, Palamà Z, Marazzato J, Bagliani G, Leonelli FM, De Ponti R. Arrhythmias in Patients with Implantable Devices. Card Electrophysiol Clin. 2019 Jun;11(2):363-373. doi: 10.1016/j.ccep.2019.02.005.

3. L. Sciarra, M. Nesti, Z. Palamà, E. Cavarretta, E. Salustri, L. Calò, P. Delise. Sport Activity in Subjects with Implantable Defibrillator in Sport Related Sudden Cardiac Death, Causes and Prevention. Springer, Cham. 2021: 184-194 doi: 10.1007/978-3-030-80447-3

4. Knops RE, Olde Nordkamp LRA, Delnoy P, et al. Subcutaneous or transvenous defibrillator therapy (PRAETORIAN trial). N Engl J Med. 2020;383:526-536.https://doi.org/10.1056/NEJMoa1915932 